\title{
Energy consumption profiles in buildings
}

\author{
A. Massaguer ${ }^{1}$, J. Meléndez $^{1}$ and S. Herraiz ${ }^{1}$ \\ ${ }^{1}$ Institute of Informatics and Applications \\ Control Engineering and Intelligent Systems (eXiT) \\ Polytechnic High School, University of Girona \\ Campus de Montilivi, 17071 Girona (Spain) \\ Phone/Fax number: +0034 972 418391, e-mail: albert.massaguer@udg.edu
}

\begin{abstract}
We propose an intelligent data analysis method for modelling and prediction of electricity consumption in buildings. The objective is to enable a building management system to be used for forecasting and detection of abnormal energy use, determining which are the consumption origins and compare them with other buildings using indicators.
\end{abstract}

\section{Key words}

Energy management, intelligent system, prediction, building energy comparison, modelling.

\section{Introduction}

Energy demand, its economic consequences and the implications for climate change are probably the most significant challenges facing any government at the start of the $21^{\text {st }}$ century. In consequence, both the industry and governments are making huge investments on the supply side to increase the share of renewable energies and at the same time reduce the carbon fingerprint, as EU directive states [1]. However, on the consumers' side, improvements on energy efficiency and awareness are also required [2]. Last EU directive on energy efficiency [3] reinforces this view and gives an exemplar role to governments and public authorities, forcing specific actions to public buildings and at the same time that demands better energy monitoring and planning strategies [4].

In addition, energy consumption of buildings in developed countries represent $20-40 \%$ of total energy use [5]. Thus, becomes essential to make available comprehensive building energy information to allow efficiently plan energy policies.

This work is aligned with these tendencies and proposes a method for analysis and identification of consumption patterns for monitoring.

\section{Objectives}

In this paper, we propose an intelligent data analysis method for modelling and prediction of daily electric consumption in buildings. The objective is to enable building management systems to be used for forecasting and detection of abnormal energy use, and besides, to offer indicators based on different consumption origins to allow building energy comparison.

First, a simplistic method is proposed to reduce the daily consumption signals into simpler and meaningful profiles.

Then a signal analysis is employed to discern, from the total daily consumption profile, each contribution of each consumption origin: base consumption, activity consumption, cleaning consumption and air conditioning consumption.

Finally, a simple classifier is used to predict the daily electricity consumption profiles. Forecasting is carried out making the summation of each consumption origin depending on the kind of the day (work, holiday...) and the expected temperature.

\section{Platform}

Girona University campus was used as the platform to obtain the power consumption patterns, Fig. 1. Each faculty was equipped with power meters that acquired data since 2010 up to now.

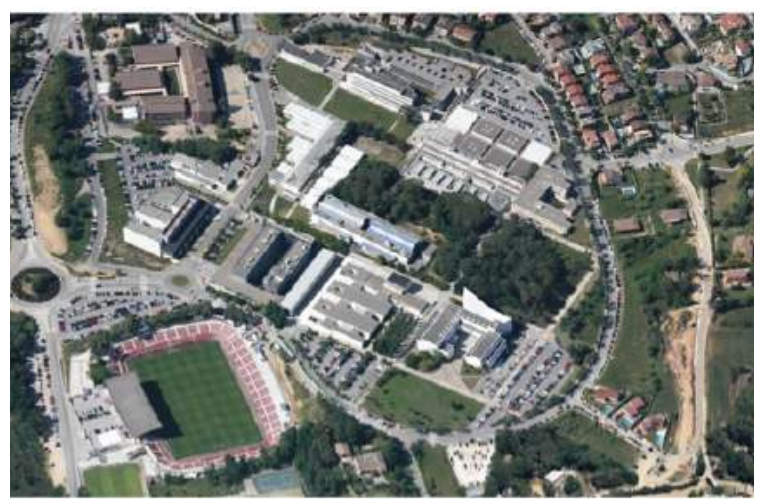

Fig. 1. Girona University campus. 
Basic information:

- Campus surface: $94,200 \mathrm{~m}^{2}$

- Buildings: 15

- Power Meters: 26

- Global Energy Consumption $/ \mathrm{m}^{2}: 78 \mathrm{kWh} / \mathrm{m}^{2} / \mathrm{year}$

- Loads: lighting, HVAC, computers, engines, lab appliances, small machinery, instrumentation.

- Typology of buildings: academic buildings / faculties (10), libraries (2), office buildings (3).

- Weather station.

- Other sensing equipment: wireless sensors.

\section{Monitored data acquisition and database}

Consumption at the different buildings has been monitored using 26 power meters that acquire data every 15 minutes and store it in a database, Fig. 2.

Apart from power meters, a weather station provides, every 5 minutes, the ambience temperature, stored also in the database.

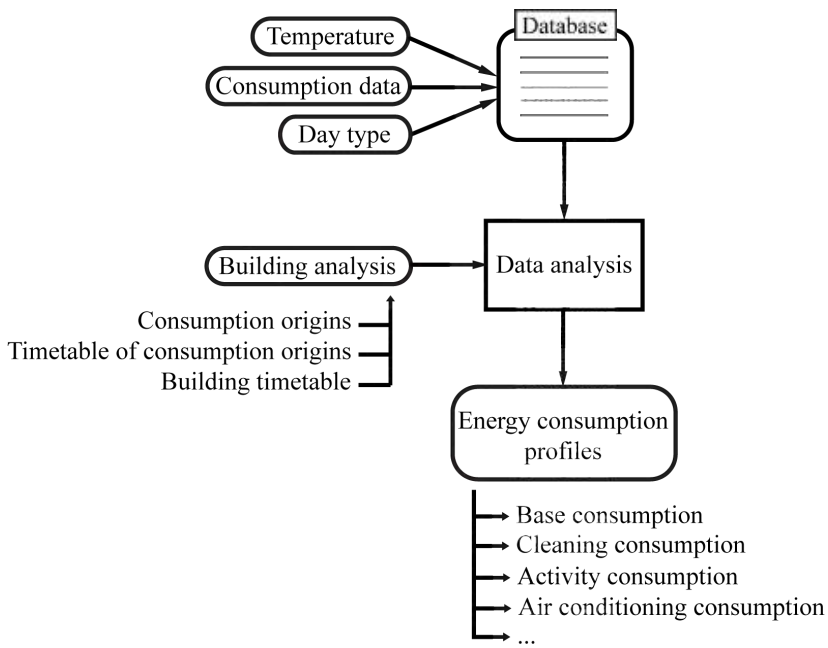

Fig. 2. The model's procedure.

Furthermore, database contains an extra variable related to kind of day. Each day is classified in holiday and working day, and each working day is divided in school day and non school day, Fig. 3.

An example of a day consumption profile can be observed in Fig. 4 (blue line)

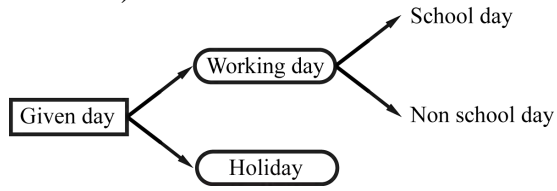

Fig. 3. Day types.

\section{Simplification method}

Data acquired is simplified, maintaining meaningful values, in order to magnify the main consumption behaviour Fig. 4.

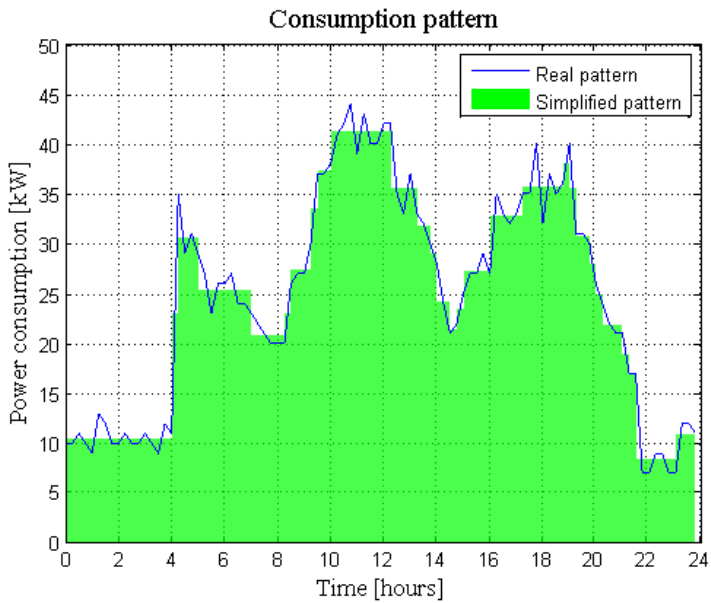

Fig. 4. Total energy consumption pattern of Communal classroom building on March $22^{\text {nd }}$.

Green area represents the simplified pattern. The objective of this reduction is to obtain a simpler and meaningful model of the real pattern in order to discriminate small oscillations and reveal the overall behaviour.

Simplification is carried out using three signal filters in series. First of them, Filter 1, consists in localizing when the power consumption increment, between two consecutive instants, exceeds a parameter $\mathrm{P}$, in $\mathrm{kW}$. Thus, pattern is discretised using instants when Filter 1 consideration is fulfilled. Evolution between these instants is defined constant, following the mean of intermediate consumption values. The resulting signal is shown in Fig. 5.

Pattern obtained is simpler than the original and follows the overall behaviour. Its simplicity increments when value $\mathrm{P}$ grows. This is due to the fact that fewer points accomplish Filter 1 criteria when $\mathrm{P}$ tends to increase.

Nevertheless, signal obtained after Filter 1 simplification can exhibit small step increments if consumption pattern oscillate rapidly with amplitude larger than P. Cases a) and $b$ ) show this behaviour for periods between 6 to 10 , 13 to 16 and 18 to 21 .

Filter 2 consists in detecting when steps of signal obtained after Filter 1 are smaller than a value $\mathrm{R}$, in $\mathrm{kW}$. When this condition is fulfilled Filter 2 deletes the point stored in Filter 1. The resulting signal is shown in Fig. 6.

Finally, Filter 3 establishes the minimum peak duration. The output is a signal with steps of length larger than $\mathrm{T}$ minutes. The resulting signal is shown in Fig. 7.

Green surface (energy) always have exactly the same value as the area below original pattern, regardless of Filter used and parameter defined. Also, area between steps contains the same energy as the original signal. The simplest pattern is always a constant area. 
a)

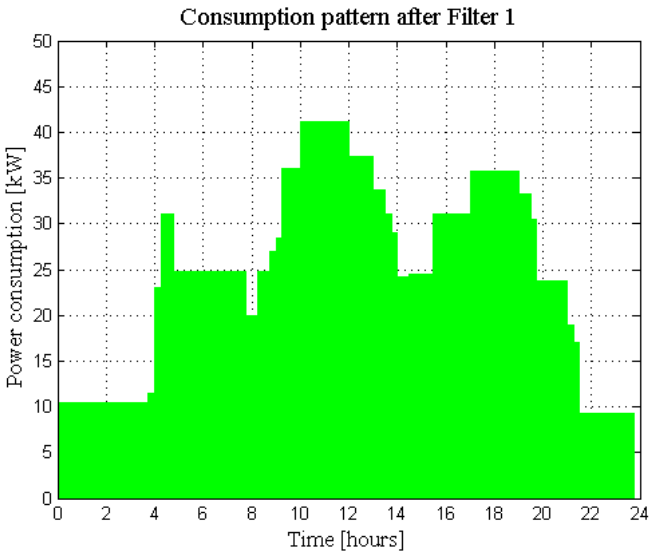

b)

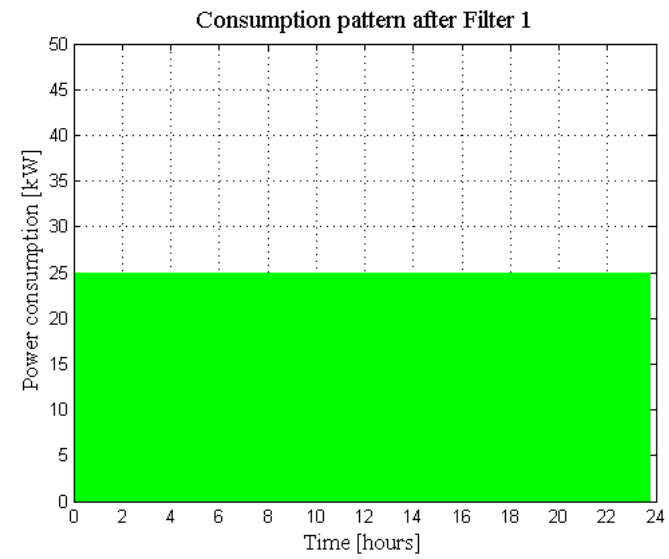

Fig. 5. Total energy consumption pattern simplification of Communal classroom building on March $22^{\text {nd }}$ using Filter 1. a) $\mathrm{P}=5 \mathrm{~kW}$. b) $\mathrm{P}=30 \mathrm{~kW}$.

a)

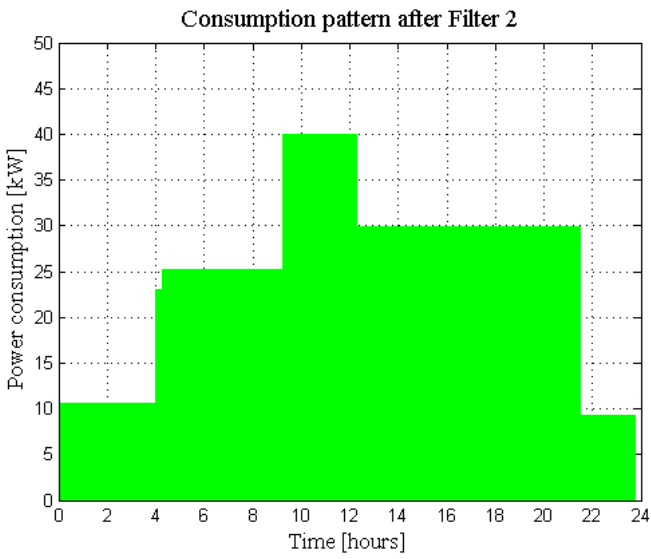

b)

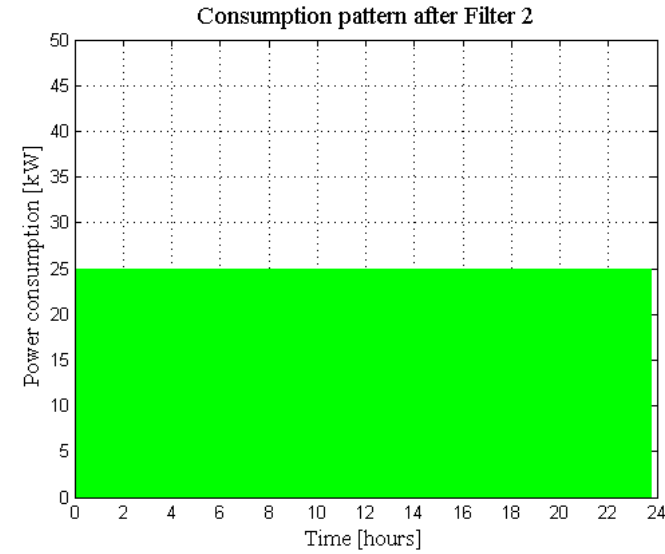

Fig. 6. Total energy consumption pattern simplification of Communal classroom building on March $22^{\text {nd }}$ using Filter $1 \& 2$. a) $\mathrm{P}=\mathrm{R}=5 \mathrm{~kW}$. b) $\mathrm{P}=\mathrm{R}=30 \mathrm{~kW}$. a)

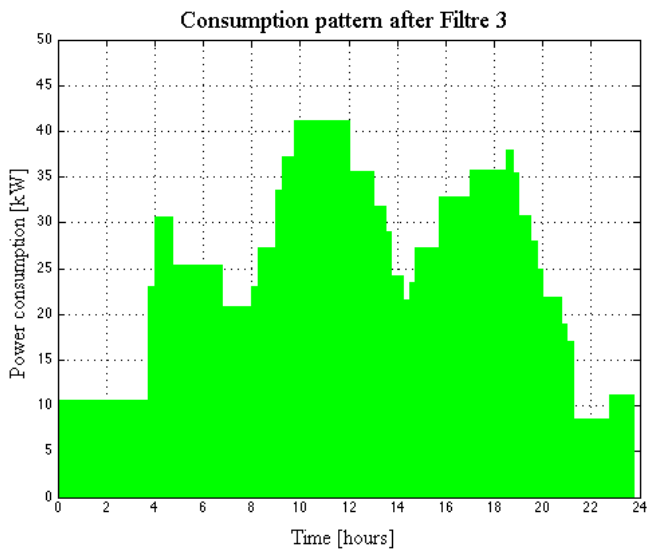

b)

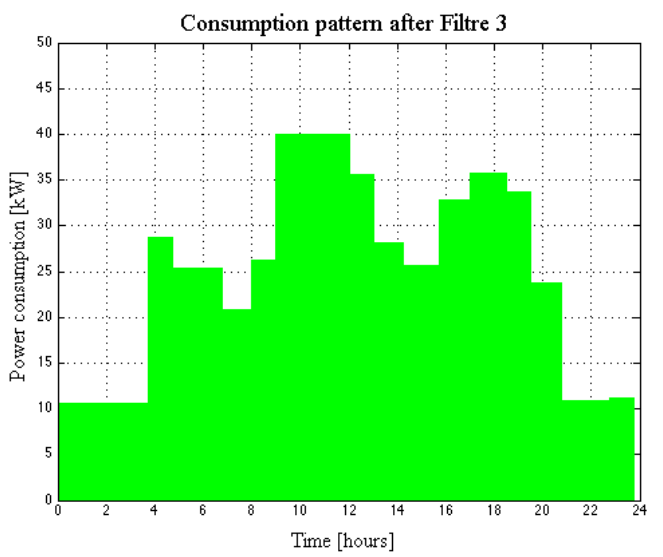

Fig. 7. Total energy consumption pattern simplification of Communal classroom building on March $22^{\text {nd }}$ using Filters 1, 2 \& 3. a) $\mathrm{P}=\mathrm{R}=2 \mathrm{~kW} \& \mathrm{~T}=15$. b) $\mathrm{P}=\mathrm{R}=2 \mathrm{~kW} \& \mathrm{~T}=60$.

Selecting the appropriate $\mathrm{R}, \mathrm{P}$ and $\mathrm{T}$ values, resultant pattern is representative of the original power consumption curve: with same peaks and valleys, maintaining its temporal evolution and keeping the same accumulated period energy, either for the whole period and between each step.

\section{Data analysis}

\section{Nomenclature}

$\boldsymbol{\alpha}$ : dimensionless consumption coefficient, scalar

$\boldsymbol{C}$ : consumption pattern, vector

$\overrightarrow{\boldsymbol{C}}$ : consumption patterns of various days, cell

$\overline{\boldsymbol{C}}$ : mean consumption pattern, scalar

$\overline{\overrightarrow{\mathbf{C}}}$ : mean of consumption patterns, vector

$\overline{\overline{\overrightarrow{\mathbf{C}}}}$ : mean of mean of base consumption patterns, scalar $\boldsymbol{\varepsilon}$ : error, scalar

Consumption curve is the addition of consumptions of multiple subsystems operating according to different uses and weather conditions. Thus, in Fig. 4, lighting is the main load between 4 and 8 in the morning when the cleaning service is working in the building. Consumption due to cleaning service is defined as $\boldsymbol{C}_{\text {Clean }}$. In addition, during academic timetable, from 8 to 21 , there are different loads acting at the same time. We distinguish 
them by activity $\boldsymbol{C}_{\boldsymbol{A c t}}$, produced by lighting and computers, and air conditioning consumption $\boldsymbol{C}_{\boldsymbol{A} \boldsymbol{C}}$.

Out of working timetable, between 22 and 4 in the morning, there is always a base consumption $\boldsymbol{C}_{\text {Base }}$, mainly due to remnant energy use like night lighting, lab machinery, or security system detection. Base load is always present and represents the minimum building consumption.

In consequence, the overall building consumption will be the summation of all particular consumptions mentioned, taking into account the error $\boldsymbol{\varepsilon}_{\boldsymbol{i}}$ committed on each prediction.

$$
\begin{aligned}
\boldsymbol{C}_{T}=\sum_{i=1}^{N} \alpha_{i} \boldsymbol{C}_{i}+\boldsymbol{\varepsilon}_{i} & \text { Eq. } 1 \\
\boldsymbol{C}_{\boldsymbol{T}}=\boldsymbol{C}_{\text {Base }}+\boldsymbol{C}_{\text {Clean }}+\boldsymbol{C}_{\text {Act }}+\boldsymbol{C}_{\text {AirCond }} & \text { Eq. } 2 \\
\boldsymbol{C}_{\text {Base }}=\boldsymbol{\alpha}_{\boldsymbol{B}} \boldsymbol{C}_{\boldsymbol{B}}+\boldsymbol{\varepsilon}_{\mathrm{B}} & \\
\boldsymbol{C}_{\text {Clean }}=\boldsymbol{\alpha}_{\boldsymbol{C}} \boldsymbol{C}_{\boldsymbol{C}}+\boldsymbol{\varepsilon}_{\text {Clean }} & \text { Eq. } 3 \\
\boldsymbol{C}_{\text {Act }}=\boldsymbol{\alpha}_{\boldsymbol{A}} \boldsymbol{C}_{\boldsymbol{A}}+\boldsymbol{\varepsilon}_{\text {Act }} & \text { Eq. } 4 \\
\boldsymbol{C}_{\text {AirCond }}=\boldsymbol{\alpha}_{\boldsymbol{A} C} \boldsymbol{C}_{\boldsymbol{A C}}+\boldsymbol{\varepsilon}_{\text {AirCond }} & \text { Eq. } 5 \\
& \text { Eq. } 6
\end{aligned}
$$

To extract each particular consumption pattern it is necessary to establish searching parameters for the stored data. These searching parameters are defined strategically to find particular consumptions when they are supposed to be isolated. For example, during working days the minimum consumption is the base one, then, knowing base consumption, looking for a non school day and subtracting base consumption to it we will obtain cleaning consumption pattern.

To improve method's accuracy we divided daily timetable into 7 time zones, $\boldsymbol{T Z}$.

$$
\begin{aligned}
& \text { TZ1 - } 0 \text { to } 4 \text { a.m. } \\
& \text { TZ2 - } 4 \text { to } 8 \text { a.m. } \\
& \text { TZ3 - } 8 \text { a.m. to } 2 \text { p.m. } \\
& \text { TZ4 - } 2 \text { to } 3 \text { p.m. } \\
& \text { TZ5 - } 3 \text { to } 6 \text { p.m. } \\
& \text { TZ6 - } 6 \text { to } 9 \text { p.m. } \\
& \text { TZ7 - } 9 \text { to } 12 \text { p.m. }
\end{aligned}
$$

Time zones are defined accordingly to daily schedule provided in database inputs. Academic timetable starts at 8 a.m. until 9 p.m., with a brake from 2 to 3 p.m.. Cleaning service schedule is from 4 to 8 a.m..

Procedure to find the base consumption on holidays is defined as follows, calculating the mean consumption of all number $\boldsymbol{N}$ holidays.

$$
\boldsymbol{C}_{B}=\boldsymbol{C}_{B H}=\frac{\sum_{i=1}^{N} \overrightarrow{\boldsymbol{C}}_{\text {Holiday }_{i}}}{N}=\overline{\overrightarrow{\boldsymbol{C}}}_{\text {Holiday }}
$$

Base consumption is supposed to be a constant value during the period as the holiday consumptions show.

It is known that base consumption for working days can vary from the one extracted from holidays, mainly because on weekdays some computers or lab machinery remain operating during night. For this reason it is necessary to define base consumption for working days $\boldsymbol{C}_{\boldsymbol{B} \boldsymbol{W}}$.

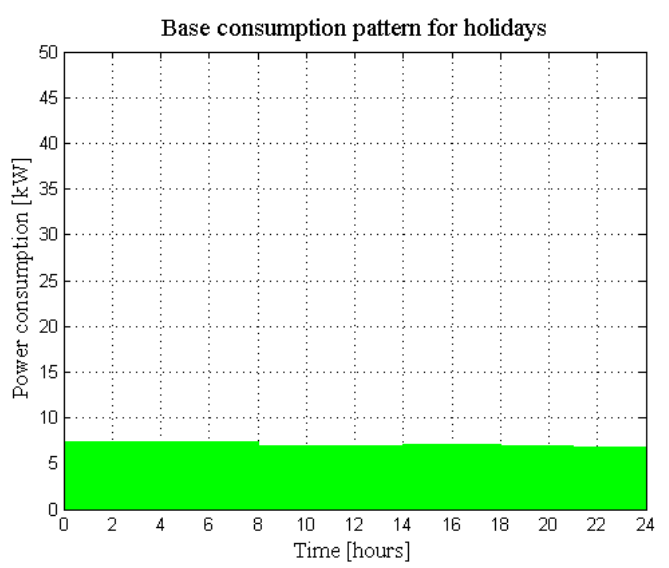

Fig. 8. Base consumption pattern for holidays.

Base consumption for working days is computed as the vectorization of minimum value of mean working day consumption for each time zone, either school and non school day, Fig. 9. In case of Communal classroom building and for the majority of docent buildings, base consumption for working days is expected to appear out of docent timetable, for example at night TZ1, Fig. 10.

$$
\boldsymbol{C}_{B W}=\operatorname{vec}\left(\min \left(\left.\overline{\boldsymbol{C}}_{\text {Working }}\right|_{T Z 1},\left.\overline{\boldsymbol{C}}_{\text {Working }}\right|_{T Z 2}, \ldots,\left.\overline{\boldsymbol{C}}_{\text {Working }}\right|_{T Z 7}\right)\right)
$$

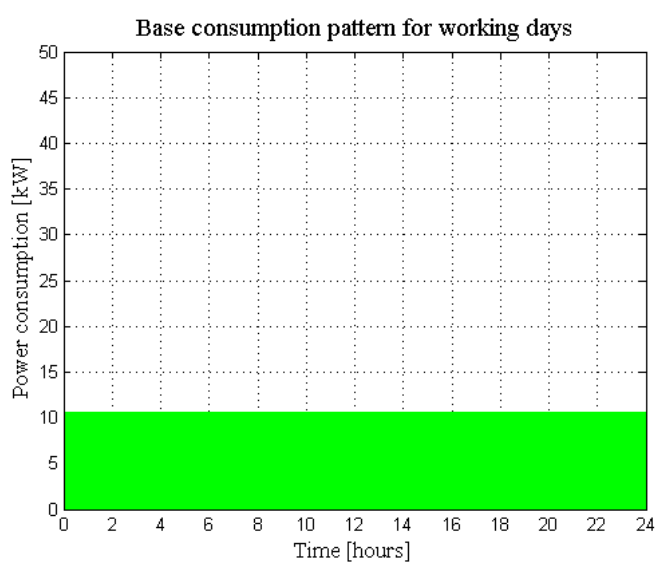

Fig. 9. Base consumption pattern for working days.

We know that cleaning service works from 4 to 8 in the morning. In this period, TZ2, consumption is due only to cleaning service and base load, Fig. 10.

Where,

$$
\mathrm{C}_{\mathrm{C}}=\overrightarrow{\mathrm{C}}_{\text {Working }_{\mathrm{i}=\boldsymbol{\beta}}}-\overline{\overrightarrow{\mathrm{C}}}_{\text {Base Working }}
$$

Eq. 9

$$
\boldsymbol{\beta}=\min \left(\left|\overline{\mathbf{C}}_{\text {Working }_{\mathbf{i}}}\right|_{\text {TZ2 }}-\left.\overline{\mathbf{C}}_{\text {Working }}\right|_{\text {TZ2 }} \mid\right) \quad \text { Eq. } 10
$$

Then, looking for the minimum consumption difference between the working loads in TZ2, green dots, and the mean of TZ2 period, green line, it is possible to find the cleaning consumption pattern. $\boldsymbol{\beta}$ is the day when Eq. 10 is fulfilled, Fig. 10. 
Thus, cleaning consumption pattern, Fig. 11, is the pattern during TZ2 at day $\boldsymbol{\beta}$, here day 151.

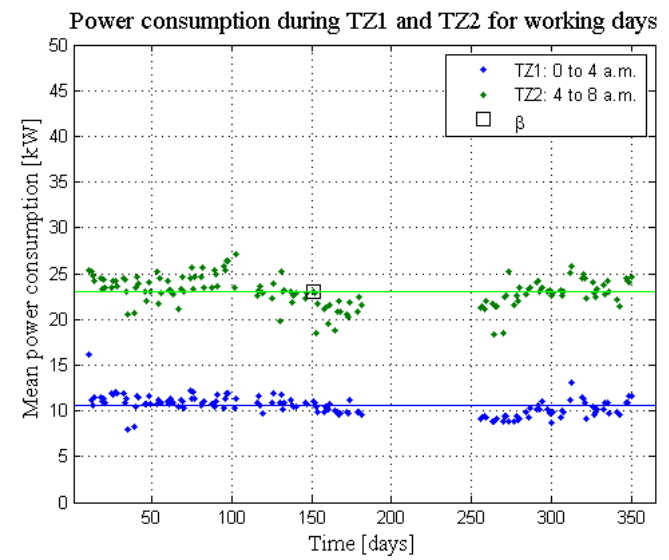

Fig. 10. Energy spent during TZ1 and TZ2 for working days.

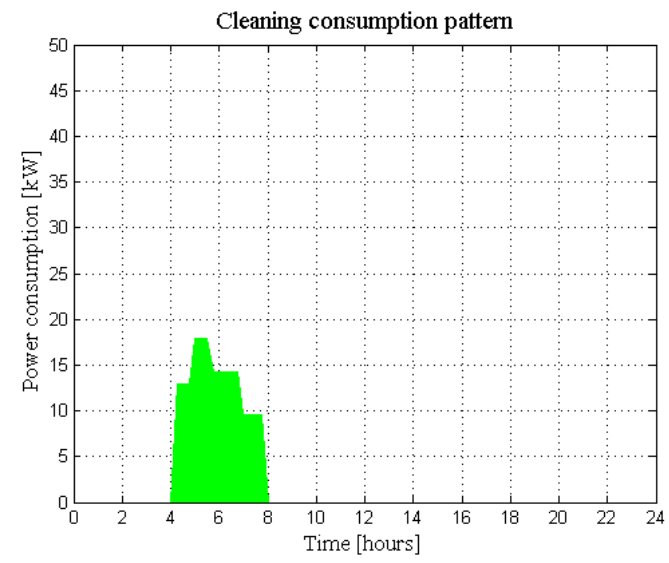

Fig. 11. Cleaning consumption pattern.

Activity consumption is dependant of the building docent timetable, generally comprised from 8 to 21 . In this period, a part from base consumption, there is to be expected to yield load due to air conditioning. To extract activity consumption from the overall pattern it is necessary to search for periods when air conditioning is not working, i.e. when the ambience temperature is similar to comfort temperature.

Then, activity consumption is computed as the mean working consumption of each time zone when mean temperature of each time zone is comprised between 24 and 26 centigrade. The overall activity consumption signal is the concatenation of activity consumption at each time zone. The resulting signal is shown in Fig. 12.

$\mathbf{C}_{\mathbf{A}}=\left(\left.\left.\left.\left.\overline{\mathbf{C}}_{\text {Working }}\right|_{\text {TZ3 }}|| \overline{\mathbf{C}}_{\text {Working }}\right|_{\text {TZ4 }}|| \overline{\mathbf{C}}_{\text {Working }}\right|_{\text {TZ5 }}|| \overline{\mathbf{C}}_{\text {Working }}\right|_{\text {TZ6 }}\right)_{24<\mathrm{T}<26}$ Eq. 11

Air condition consumption $\mathbf{C}_{\mathbf{A C}}$ is the remaining load origin that allows defining completely the total consumption pattern of the building. However, $\mathbf{C}_{\mathrm{AC}}$ is not a constant value, and it depends on the day and temperature, Fig. 13.

Consequently, it is necessary to obtain the relationship between temperature and consumption. It is carried out with two data fittings; one for temperatures lower than comfort temperature, heating process or cold consumption, and the remaining one for higher temperatures, refrigeration process or hot consumption.

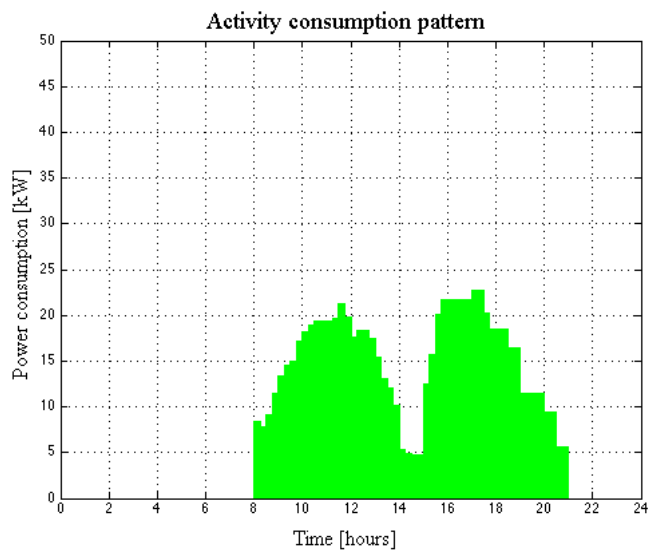

Fig. 12. Activity consumption pattern.

The reason of this differentiation is the slope's value. We expect to obtain negative slope for heating, and a positive one for cooling. In Fig. 13, this behaviour is not obtained because in Communal classroom building, periods of cooling coincide with summer vacations.

Then, knowing the relationship between temperature and consumption we define a base point, $\boldsymbol{C}_{\boldsymbol{A} C}$, of which we will obtain the air consumption base pattern, shape pattern. This base pattern will be useful for consumption forecasting because it will increase or decrease its value in function of temperature using $\boldsymbol{\alpha}_{\mathbf{A C}}$. This dependency is modelled with dimensionless consumption coefficients Eq. 25.

Base point is found searching the closest consumption point to the fitting curve, for heating and cooling cases. They are represented with a square in Fig. 13.

$$
\begin{aligned}
\mathrm{C}_{\mathrm{AC}} & =\mathrm{C}_{\text {Working }_{\mathrm{i}=\gamma}} \\
\gamma & =\min \left(\frac{\left|\mathrm{ax}_{\mathrm{i}}+\mathbf{b y}_{\mathrm{i}}+\mathbf{c}\right|}{\sqrt{\mathbf{a}^{2}+\mathbf{b}^{2}}}\right)
\end{aligned}
$$

$\gamma$ is the day when Eq. 13 is fulfilled, Fig. 10. And,

$$
\begin{array}{rlr}
\mathbf{x}_{\mathbf{i}} & =\mathbf{T}_{\mathbf{i}} & \text { Eq. } 14 \\
\mathbf{y}_{\mathbf{i}} & =\mathbf{C}_{\text {Working }_{\mathbf{i}}} & \text { Eq. } 15 \\
\mathbf{C}_{\text {Working }_{\mathbf{i}}} & =-\mathbf{a T}_{\mathbf{i}}-\mathbf{c} & \text { Eq. } 16
\end{array}
$$

$a$, the slope, and $c$, the y-intercept, are the constant parameters of the linear equation of fitting curve. They can be found carrying out a data fitting for cold and hot consumptions Fig. 13. The dimensionless consumption coefficients scale the strength of each consumption origin at a particular temperature and kind of day, Fig. 3.

$$
\begin{aligned}
\left.\alpha_{B}\right|_{\text {Holiday }} & =1 \\
\left.\alpha_{B}\right|_{\text {Working, non school day }} & =1 \\
\left.\alpha_{B}\right|_{\text {Working, school day }} & =\frac{\overline{\overline{\boldsymbol{C}}}_{B W}}{\overline{\overline{\overrightarrow{\boldsymbol{C}}}}_{B H}}
\end{aligned}
$$




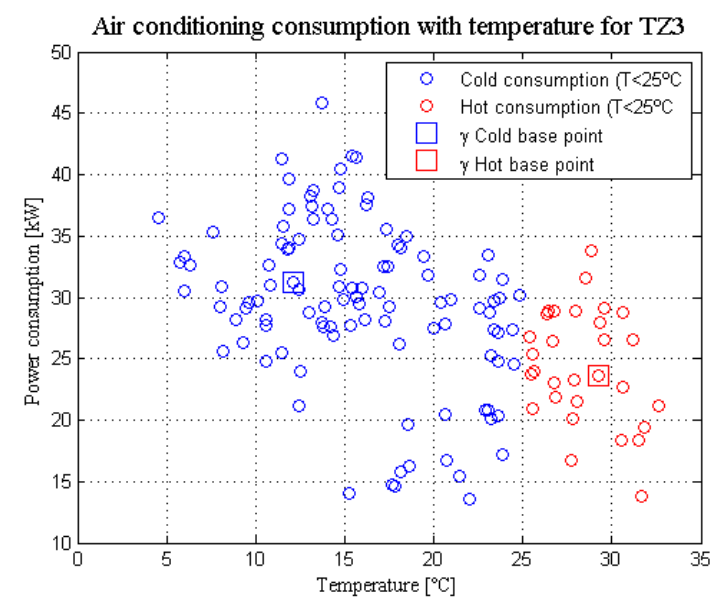

Fig. 13. Air conditioning consumption evolution of school days with temperature for TZ3.

$\boldsymbol{C}_{\boldsymbol{B} \boldsymbol{H}}$ and $\boldsymbol{C}_{\boldsymbol{B} \boldsymbol{W}}$ correspond to the minimum daily consumptions for a holiday and a working day respectively. These two signals are considered constants during each day and period.

Cleaning is a daily activity in educational buildings and its consumption pattern respond to a regular timetable, usually out of school schedule and in workday.

$$
\begin{gathered}
\left.\alpha_{\text {Cleaning }}\right|_{\text {Holiday }}=0 \\
\left.\alpha_{\text {Cleaning }}\right|_{\text {Working }}=1
\end{gathered}
$$

Load due to the activity developed in the building depends on the day type, being present only if it's a working day.

$$
\begin{aligned}
\left.\alpha_{\text {Act }}\right|_{\text {Holiday }} & =0 \\
\left.\alpha_{\text {Act }}\right|_{\text {Working, school day }} & =1
\end{aligned}
$$

Finally, air conditioning is an important consumption in most of the buildings, varying its value with the temperature $\boldsymbol{T}$ and the day type.

$$
\begin{aligned}
\left.\alpha_{A C}\right|_{\text {Holiday }} & =0 \\
\left.\alpha_{A C}\right|_{\text {Working, school day }} & =\frac{a T+\left.b\right|_{\text {Predicted Point }}}{a T+\left.b\right|_{\text {Base Point }}}
\end{aligned}
$$

Predicted point is the consumption expected considering a temperature $T$.

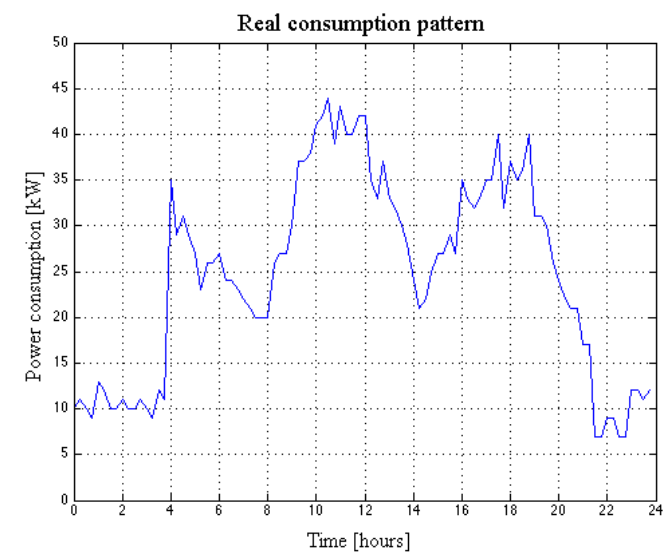

Fig. 14. Real consumption pattern.
Finally, considering all consumption origins and making a summation of all of them we obtain the reconstructed pattern, Fig. 15.

Reconstructed pattern exhibits similar overall behaviour to original one. Also presents the same number of peaks and valleys, capturing the energy consumption variation along the period. However, reconstructed pattern show little discrepancy in front of the original signal, mainly due to the simplification and the supposition of constant patterns along the period. The difference between them is $7,26 \%$.

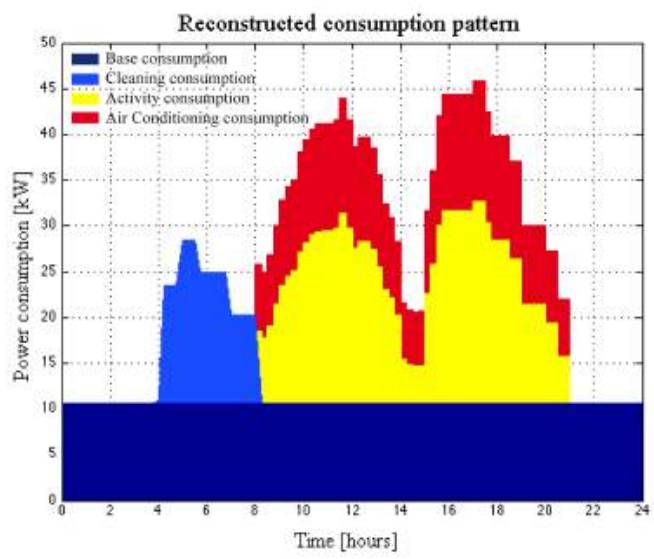

Fig. 15. Total consumption pattern reconstruction from total consumption data.

A quantification of the error committed is carried out.

Table I. - Error quantification.

\begin{tabular}{|l|c|c|c|}
\hline \multicolumn{1}{|c|}{ Consumption } & $\begin{array}{c}\text { Mean } \mu \\
{[\mathrm{kW}]}\end{array}$ & $\begin{array}{c}\text { Std. dev. } \sigma \\
{[\mathrm{kW}]}\end{array}$ & Std. error \\
\hline Base & 7,11 & 1,73 & 0,06 \\
\hline Cleaning & 13,56 & 2,05 & 0,17 \\
\hline Activity & 15,36 & 2,79 & 0,99 \\
\hline Air Conditioning & 24,17 & 6,18 & 6,18 \\
\hline
\end{tabular}

Air-conditioning, as seen in Fig. 13 and Table I, exhibit high variability and error, which denotes that this consumption is not dependent only on temperature. Further work will focus on considering Day Degrees for better data fitting.

\section{Main contributions}

Energy efficiency analysis methodologies require software knowledge, large amount of time involved on gathering power information of lights or devices, and to capture power consumption patterns from particular circuits to know its origin.

Data analysis described needs only the global consumption pattern of the building for as much days as possible, ambience temperature of these days, and a day classification. With these simple values, method is capable to distil consumption origins of a given building. Knowing them, it is possible to predict future consumptions, and, derived from this, to detect abnormal 
energy use if the current consumption differs from the expected one.

In addition, knowing the consumption origins of the building it is feasible to distinguish if there are abnormalities on each of them just comparing it with other buildings.

Finally, comparison between building consumptions can be used as indicators of energy efficiency.

\section{References}

[1] Directive of the European parliament and of the council on the promotion of the use of energy from renewable sources (2009/28/EC).

[2] Enerficiency project. ITEA 210009.

[3] Directive of the European parliament and of the council on energy efficiency (2012/27/EU).

[4] The International Organization for Standardization (ISO). Win the energy challenge with ISO 50001; June 2011.

[5] L. Prez-Lombard, J. Ortiz \& C. Pout, A review on buildings energy consumption information, Energy and Buildings, 2008;40(3):394-398. 